\title{
Controlling the Nonequilibrium Interlayer Exchange Coupling in Asymmetric Magnetic Tunnel Junctions
}

\author{
Y.-H. Tang, ${ }^{1}$ Nicholas Kioussis, ${ }^{1}$ Alan Kalitsov, ${ }^{1}$ W. H. Butler, ${ }^{2}$ and Roberto $\mathrm{Car}^{3}$ \\ ${ }^{1}$ Department of Physics, California State University, Northridge, California 91330-8268, USA \\ ${ }^{2}$ MINT Center, University of Alabama, P. O. Box 870209, Tuscaloosa, Alabama, USA \\ ${ }^{3}$ Department of Chemistry, Princeton University, Princeton, New Jersey 08544-0001, USA
}

(Received 7 April 2009; published 31 July 2009)

\begin{abstract}
We predict an oscillatory bias behavior of the fieldlike spin torque, $T_{\perp}$, in magnetic tunnel junctions, which can be selectively controlled via the asymmetry in band filling between the ferromagnetic leads. This can lead to a linear or quadratic low-bias behavior, including tuning the bias-induced reversal of $T_{\perp}$. These findings reconcile the apparently contradictory experimental results recently reported in the literature. The underlying mechanism for the nonequilibrium interlayer exchange coupling (IEC) of noncollinear configurations is the interplay of four independent IEC for the majority- and minority-spin bands of the leads solely in the ferromagnetic configuration.
\end{abstract}

DOI: 10.1103/PhysRevLett.103.057206

PACS numbers: 85.75.- d, 72.25.-b, 73.40.Gk

The current-induced magnetization reversal (CIMR) in magnetic tunnel junctions (MTJ) via the so-called spintransfer torque (STT), originally predicted by Slonczewski [1] and Berger [2], has attracted intensive investigations both experimentally [3-7] and theoretically [8-11]. The CIMR offers the promise for making nonvolatile magnetoresistive random access memory devices (MRAM), in which information is written using the STT effect rather than the field-induced magnetic switching.

The spin torque can be decomposed into a fieldlike, $T_{\perp}$, and a spin transfer, $T_{\|}$, components both orthogonal to the magnetization of the free ferromagnetic (FM) lead, where the first (latter) are perpendicular (parallel) to the plane of the magnetizations of the left and right FM leads, but with different bias behavior. For example, while recent experiments indicate [5-7] that $T_{\|}$reverses sign on changing the current direction, we have recently predicted an anomalous bias behavior, where $T_{\|}$can exhibit a sign reversal without a corresponding sign reversal of the bias or even a quadratic bias dependence [8]. Unlike spin valves, where $T_{\perp} \ll T_{\|}, T_{\perp} \approx T_{\|}$in MTJ, thus playing also an important role in the CIMR [5-7].

The bias behavior of $T_{\perp}$, directly related to the nonequilibrium interlayer exchange coupling (IEC), remains unresolved and controversial. On the theoretical side, the pioneering work of Slonczewski [1] showed that for symmetric MTJ at zero bias, $T_{\perp}$ can change sign with decreasing potential-barrier height. Recent calculations $[8,10,12]$ have predicted a purely quadratic bias dependence of $T_{\perp}$ in symmetric MTJ, with $d^{2} T_{\perp}(V) / d V^{2}<0$ for any value of band filling (BF) and exchange interaction. On the experimental side, Sankey et al. [5] and Kubota et al. [6] have measured recently the bias dependence of $T_{\perp}$ in $\mathrm{Co}_{60} \mathrm{Fe}_{20} \mathrm{~B}_{20} / \mathrm{MgO} / \mathrm{Co}_{60} \mathrm{Fe}_{20} \mathrm{~B}_{20}$ MTJ, where the thickness of the free FM layer and the barrier are slightly different. They both find a quadratic bias dependence of
$T_{\perp}$ in agreement with theoretical calculations [8-10], but with an apparently different bias curvature, presumably due to the different sign convention employed for the direction of both components of the STT. On the other hand, Petit et al. [7] observed a linear bias dependence of $T_{\perp}$ in a $\mathrm{CoFe} / \mathrm{Al}_{2} \mathrm{O}_{3} / \mathrm{CoFe}$ MTJ. Thus, despite its importance in CIMR applications, the bias behavior of $T_{\perp}$ remains unresolved.

The purpose of this Letter is to understand the underlying electronic mechanism that controls the bias behavior of $T_{\perp}$, and reconcile the origin of the experimental controversies, without invoking the recently proposed inelastic effects [13]. We predict that $T_{\perp}$ oscillates with bias which can be tuned via the MTJ asymmetry. We find an interesting low-bias behavior of IEC, ranging from linear to quadratic bias dependence, with positive or negative bias curvature, including tuning the bias-induced reversal of $T_{\perp}$. We derive a novel general expression relating the bias behavior of $T_{\perp}$ in noncollinear MTJ with that of collinear [FM and antiferromagnetic (AFM)] configurations, independent of the details of the electronic structure. We demonstrate that the wide range of bias behavior of IEC in noncollinear MTJ can be understood by the interplay of four independent IEC, $J_{\mathrm{FM}}^{\sigma \sigma^{\prime}}$ associated with the majority- and minority-spin channels, $\sigma, \sigma^{\prime}=\uparrow, \downarrow$, of the two leads solely in the FM configuration. The bias dependence of the IEC components can be selectively tuned with the BF of the free and pinned FM layers, thus opening a new avenue for controlling experimentally $T_{\perp}$.

The magnetization of the right, $\mathbf{M}_{\mathbf{R}}$, and left, $\mathbf{M}_{\mathbf{L}}$, semiinfinite FM leads sandwiching the 5-layer nonmagnetic insulating barrier (B), are along the $z$ and $x$ directions, respectively, as shown in Fig. 1. The $y$ direction is normal to the FM/B interface ( $x-z$ plane). Under a rotation operation of $\mathbf{M}_{\mathbf{L}}$ along the direction of $\mathbf{M}_{\mathbf{R}}$ the majority(minority-) energy bands of the left lead acquire an addi- 


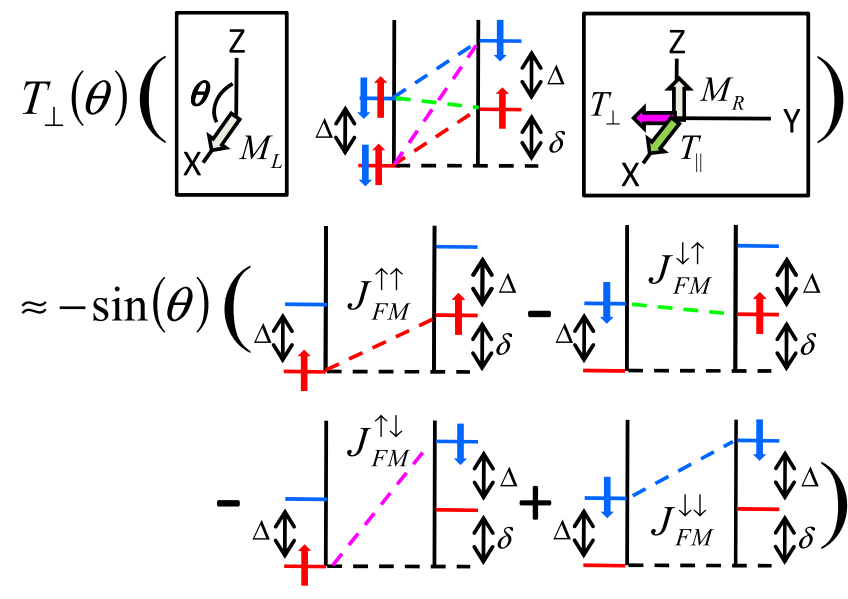

FIG. 1 (color online). Schematic of asymmetric MTJ, where the magnetization, $\mathbf{M}_{\mathbf{R}}$, of the right FM lead is along $z$, while that of the left FM lead, $\mathbf{M}_{\mathbf{L}}$, is rotated by the angle $\theta$ around the $y$ axis with respect to $\mathbf{M}_{\mathbf{R}}$. The asymmetry energy parameter and the exchange spin splitting are denoted by $\delta$ and $\Delta$, respectively. The red and blue horizontal solid lines represent the bottom of the majority- and minority-spin energy bands, respectively. Upon rotation of $\mathbf{M}_{\mathbf{L}}$ along $\mathbf{M}_{\mathbf{R}}$, the majority- (minority-) energy bands of the left lead acquire minority- (majority-) energy contributions. The noncollinear, $T_{\perp}(\theta)$, can be expressed in terms of four independent nonequilibrium IEC, $J_{\mathrm{FM}}^{\uparrow \uparrow}, J_{\mathrm{FM}}^{\downarrow \downarrow}, J_{\mathrm{FM}}^{\uparrow \downarrow}$, and $J_{\mathrm{FM}}^{\downarrow \uparrow}$, solely in the FM configuration. The AFM configuration can be obtained by exchanging the majority and minority bands of the left lead.

tional minority- (majority-) energy band contribution, shown with the blue (red) arrows in the left lead in Fig. 1. We employ the single-orbital simple-cubic tightbinding (TB) method and the nonequilibrium Keldysh formalism to determine the nonequilibrium $T_{\perp}$. The TB nearest-neighbor spin-independent hopping matrix element, $t=-0.4 \mathrm{eV}$, in all regions and the spinindependent on-site energy of the barrier is $\varepsilon_{B}=5.4 \mathrm{eV}$. The choice of parameters provides a realistic choice for systems based on magnetic transition metals and their alloys [12]. The MTJ asymmetry in BF is introduced by rigidly shifting the majority- and minority-spin on-site energies, $\varepsilon_{R}^{\sigma}$, of the right FM lead by the asymmetry energy parameter, $\delta=\varepsilon_{R}^{\uparrow(\downarrow)}-\varepsilon_{L}^{\uparrow(L)}$, relative to those of the left FM lead, where $\varepsilon_{L}^{\uparrow}=0.6 \mathrm{eV}$ and $\varepsilon_{L}^{\downarrow}=1.2 \mathrm{eV}$. Experimentally, this could be achieved through alloying $\mathrm{Fe}(\mathrm{Co})$ with impurities which can fill up the majority $\Delta_{1}$ band. In order to minimize the model parameters, we use the same exchange splitting, $\Delta=\varepsilon_{L(R)}^{\downarrow}-\varepsilon_{L(R)}^{\uparrow}$, for both leads, shown schematically in Fig. 1. The effect of external bias, $V$, is to shift the chemical potential of the right lead with respect to that of the left lead, $\mu_{R}-\mu_{L}=e V$, where $\mu_{L}$ is fixed at the Fermi energy, $E_{F}=0 \mathrm{eV}$.

The net fieldlike spin torque on the right FM lead per interfacial unit area, $\square$, is the sum of the local spin torques, $T_{\perp, i}$, which can be simply expressed as the $y$ component of the spin-current density at the right $\mathrm{B} / \mathrm{FM}$ interface $[8,12]$

$$
T_{\perp}=\frac{t}{16 \pi^{3}} \int \operatorname{Tr}\left[\left(\hat{G}_{\alpha^{\prime}, b}^{<}-\hat{G}_{b, \alpha^{\prime}}^{<}\right) \sigma_{y}\right] d E d \mathbf{k}_{\|} .
$$

Here, $\hat{G}^{<}$is the $2 \times 2$ Keldysh Green's function matrix in spin space, the subscripts $b$ and $\alpha^{\prime}$ refer to the last site inside the barrier and the first site in the right FM electrode, respectively, $\sigma_{y}$ is the $y$-component of the Pauli matrix, $\mathbf{k}_{\|}$ is the transverse component of the wave vector, and the energy integral is over occupied states. Because of the rapid decay of $T_{\perp, i}$ from the $\mathrm{B} / \mathrm{FM}$ interface [14], the net torque for a finite FM lead of thickness $>4 \mathrm{~nm}$ is about the same as that for a semi-infinite lead.

The Keldysh Green's function matrix in Eq. (1) can be expressed as

$$
\begin{aligned}
\hat{G}_{\alpha^{\prime}, b}^{<}-\hat{G}_{b, \alpha^{\prime}}^{<}= & t^{3}\left(\hat{D}^{r} \hat{D}^{a}\right)^{-1} \hat{g}_{b a} \hat{g}_{a b}\left[\hat{g}_{R}^{r} \hat{g}_{L}^{<}+\hat{g}_{R}^{<} \hat{g}_{L}^{a}\right. \\
& \left.-\hat{g}_{L}^{<} \hat{g}_{R}^{a}-\hat{g}_{L}^{r} \hat{g}_{R}^{<}\right],
\end{aligned}
$$

where $\hat{g}_{L(R)}^{<}=f_{L(R)}\left[\hat{g}_{L(R)}^{a}-\hat{g}_{L(R)}^{r}\right]$, and $\hat{g}_{L(R)}^{r(a)}$ is the retarded (advanced) surface Green's function matrix of the isolated semi-infinite left (right) lead quantized along $\mathbf{M}_{\mathbf{R}}$. $f_{L}(E)$ and $f_{R}(E-e V)$ are the Fermi-Dirac distribution functions of the left and right leads, respectively, where $V$ is the external bias, $\hat{g}_{i j}=g_{i j} \hat{I}$ is the Green's function matrix of the isolated barrier which is real, $\hat{I}$ is the $2 \times 2$ unit matrix, and the sites $i, j=a, b$ refer to the first and last sites in the barrier. $\hat{D}^{r(a)}=\left[\left(\hat{I}-t^{2} \hat{g}_{L}^{r(a)} \hat{g}_{a a}\right) \times\right.$ $\left.\left(\hat{I}-t^{2} \hat{g}_{R}^{r(a)} \hat{g}_{b b}\right)-t^{4} \hat{g}_{a b} \hat{g}_{b a} \hat{g}_{L}^{r(a)} \hat{g}_{R}^{r(a)}\right]$, and $\left[\hat{D}^{r} \hat{D}^{a}\right] \sim \hat{I}$ in the limit of thick barrier. Substituting Eq. (2) into Eq. (1), $T_{\perp}$ can be expressed as

$$
T_{\perp} \approx-\sin \theta\left[J_{\mathrm{FM}}^{\Uparrow \uparrow}+J_{\mathrm{FM}}^{\Downarrow \downarrow}-J_{\mathrm{FM}}^{\downarrow \dagger}-J_{\mathrm{FM}}^{\Uparrow \downarrow}\right],
$$

where $\theta$ is the angle between $\mathbf{M}_{\mathbf{L}}$ and $\mathbf{M}_{\mathbf{R}}$. The nonequilibrium IEC, $J_{\mathrm{FM}}^{\sigma \sigma^{\prime}}$, between the $\sigma$ - and $\sigma^{\prime}$-spin states in the left and right leads, respectively, in the FM configuration are given by

$$
\begin{aligned}
-J_{\mathrm{FM}}^{\sigma \sigma^{\prime}}= & \frac{t^{4}}{8 \pi^{3}} \int g_{b a} g_{a b}\left[f_{L}(E) \operatorname{Im}\left\{g_{L}^{r, \sigma \sigma}\right\} \operatorname{Re}\left\{g_{R}^{r, \sigma^{\prime} \sigma^{\prime}}\right\}\right. \\
& \left.+f_{R}(E-e V) \operatorname{Re}\left\{g_{L}^{r, \sigma \sigma}\right\} \operatorname{Im}\left\{g_{R}^{r, \sigma^{\prime} \sigma^{\prime}}\right\}\right] d \mathbf{k}_{\|} d E .
\end{aligned}
$$

Equations (3) and (4) are general and independent of the electronic structure of the FM leads, and demonstrate for the first time that the bias dependence of $T_{\perp}$ in the noncollinear configuration can be expressed in terms of four independent nonequilibrium IEC solely in the FM $(\theta=0)$ collinear configuration. Our numerical results show that $J_{\mathrm{FM}}^{\sigma \sigma^{\prime}}$ are independent of the remaining spin states, $\bar{\sigma}$ and $\bar{\sigma}^{\prime}$, of the left and right leads, which therefore can be removed from the calculation by placing them high in energy. Thus, as shown in Fig. $1, J_{\mathrm{FM}}^{\Uparrow \Uparrow}=J_{\mathrm{AFM}}^{\downarrow \uparrow}, J_{\mathrm{FM}}^{\llbracket \Uparrow}=$ $J_{\mathrm{AFM}}^{\Uparrow \uparrow}, J_{\mathrm{FM}}^{\uparrow \downarrow}=J_{\mathrm{AFM}}^{\downarrow}$, and $J_{\mathrm{FM}}^{\Downarrow}=J_{\mathrm{AFM}}^{\uparrow \downarrow}$.

We first examine the effect of BF asymmetry, $\delta$, on the zero-bias behavior of $T_{\perp}(\theta=\pi / 2)$. The two terms of the 
integrand in Eq. (4), proportional to $f_{L}(E)$ and $f_{R}(E-$ $e V)$, represent the left $(L)$ and right $(R)$ reservoir contributions, $T_{\perp}^{L}$ and $T_{\perp}^{R}$, to the total $T_{\perp}=T_{\perp}^{L+R}=T_{\perp}^{L}+T_{\perp}^{R}$, respectively. In Fig. 2(a) we present the energy dependence of $T_{\perp}^{L}(E), T_{\perp}^{R}(E)$, and $T_{\perp}(E)$ (integrated over $\mathbf{k}_{\|}$) for $\delta=$ $0.0 \mathrm{eV}$ and $0.6 \mathrm{eV}$. Since $T_{\perp}$ is directly related [15] to the net IEC, $J=[E(\mathrm{FM})-E(\mathrm{AFM})] / 2$, where $E(\mathrm{FM})$ and $E(\mathrm{AFM})$ are the total energies of the FM and AFM configuration, respectively, the entire energy spectrum of occupied states contributes to the IEC. For symmetric MTJ and zero bias, $T_{\perp}^{L}(E)=T_{\perp}^{R}(E)$ exhibit a broad positive peak at about $-0.1 \mathrm{eV}$. On the other hand, for $\delta=$ $0.6 \mathrm{eV}, T_{\perp}(E)$ develops a wide negative dip around $-0.5 \mathrm{eV}$, whose origin lies primarily on the dramatic change of $T_{\perp}^{R}(E)$, which becomes negative over the entire occupied energy spectrum. This in turn changes $T_{\perp}(V=$ 0 ) from $0.2 \mathrm{peV} / \square$ for $\delta=0$ to $-0.1 \mathrm{peV} / \square$ for $\delta=$ $0.6 \mathrm{eV}$, shown in Fig. 3(a). In Fig. 2(b) we display the zerobias $\mathbf{k}_{\|}$-resolved $T_{\perp}$ integrated over occupied states for $\delta=0.0$ and $0.6 \mathrm{eV}$. For symmetric MTJ $T_{\perp}^{L}=T_{\perp}^{R}$ are positive in a narrow ring around the $\Gamma$ point and become negative around $\mathbf{k}_{\|} \sim \pi / 2$. For $\delta=0.6 \mathrm{eV}, T_{\perp}^{L}$ becomes more positive in a smaller area around the $\Gamma$ point, while $T_{\perp}^{R}$ changes to negative in a wide area around $\mathbf{k}_{\|}=0$. These results show that $T_{\perp}(V=0)$ can be tuned via the $\mathrm{BF}$ asymmetry between the $L$ and $R$ FM leads, due to the different $\mathbf{k}_{\|}$and energy dependence of $T_{\perp}^{L}$ and $T_{\perp}^{R}$.

We next examine the low-bias $(|V| \leq 0.4 \mathrm{~V})$ behavior of $T_{\perp}$, which has also been investigated experimentally. In Fig. 3(a) we present $T_{\perp}(\theta=\pi / 2)$ versus bias for various values of the asymmetry energy parameter, $\delta$. The solid points and curves denote $T_{\perp}$ calculated from Eqs. (1) and
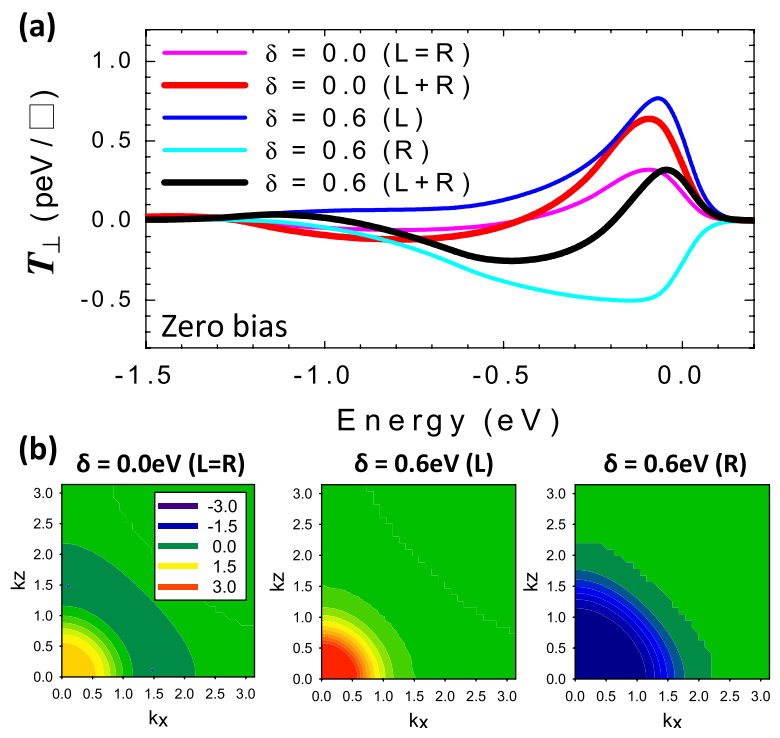

FIG. 2 (color online). (a) Energy dependence of the $L$ and $R$ contributions, $T_{\perp}^{L}$ and $T_{\perp}^{R}$, for $\theta=\pi / 2$, to the total $T_{\perp}=$ $T_{\perp}^{L+R}=T_{\perp}^{L}+T_{\perp}^{\bar{R}}$, at zero bias for $\delta=0.0$ and $0.6 \mathrm{eV}$. (b) $\mathbf{k}_{\|}$-resolved $L$ and $R$ contributions, $T_{\perp}^{L(R)}$, integrated over occupied states, for $\delta=0.0 \mathrm{eV}$ and $\delta=0.6 \mathrm{eV}$, respectively.
(3), respectively, demonstrating the excellent agreement between these two computational schemes. The most striking and unusual feature is the dramatic change of the bias behavior of $T_{\perp}$ with asymmetry, which in the low-bias limit can vary from purely quadratic dependence with negative bias curvature for the symmetric case $(\delta=$ $0.0 \mathrm{eV}$ ), to linear with negative slope for $\delta=0.6 \mathrm{eV}$, and quadratic dependence with positive bias curvature for $\delta=0.9 \mathrm{eV}$. These results reconcile the apparently contradictory experimental results in Refs. [5-7]. Furthermore, the asymmetry has a large effect both on the zero-bias $T_{\perp}$ and the bias-induced reversal of the IEC. Thus, the BF asymmetry between the left and right leads may have important practical implications on controlling the bias behavior of the IEC, which may bring a new aspect in the writing process in MRAM.

Since Eq. (3) indicates that $T_{\perp}(V)$ is determined by the interplay of the four IEC in the FM configuration, we display in Fig. 3(b) the low-bias behavior of $-J_{\mathrm{FM}}^{\sigma \sigma^{\prime}}(\sigma$, $\left.\sigma^{\prime}=\uparrow, \downarrow\right)$ for symmetric $(\delta=0.0 \mathrm{eV})$ MTJ. Both $-J_{\mathrm{FM}}^{\uparrow}$ and $-J_{\mathrm{FM}}^{\Perp}$ exhibit quadratic bias behavior with maximum
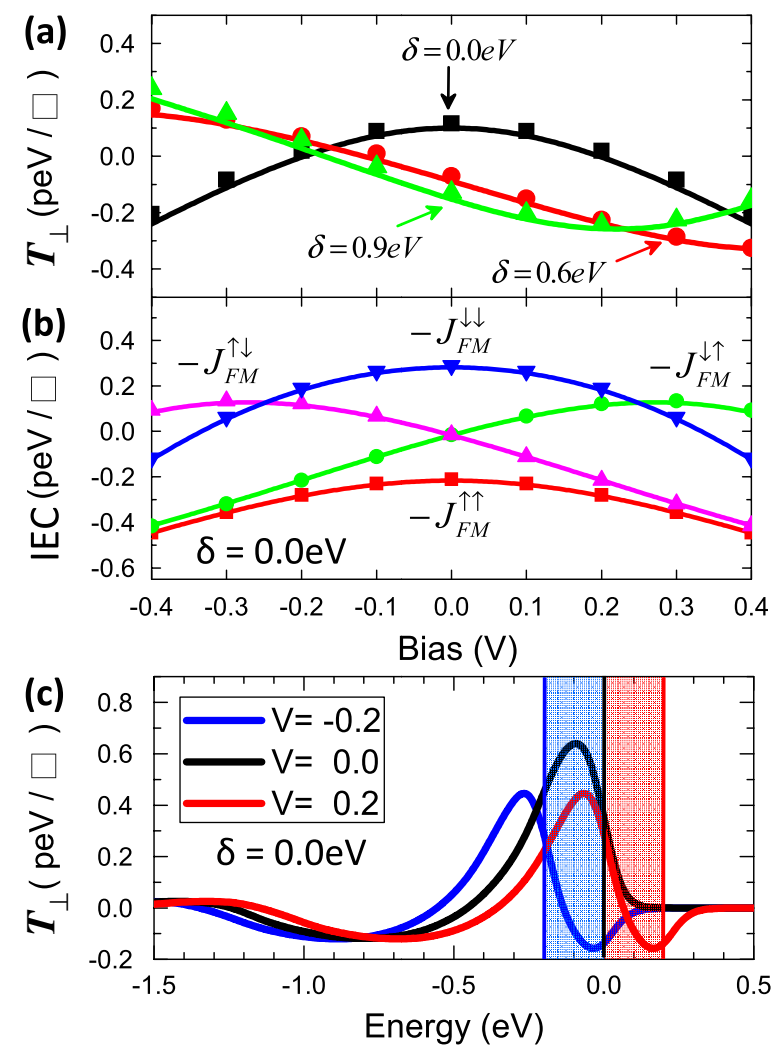

FIG. 3 (color online). (a) Low-bias $(|V| \leq 0.4 \mathrm{~V})$ behavior of $T_{\perp}(\theta=\pi / 2)$ with $\varepsilon_{L}^{\uparrow}=0.6 \mathrm{eV}$ and $\varepsilon_{L}^{\downarrow}=1.2 \mathrm{eV}$ and for $\delta=$ $0.0,0.6$, and $0.9 \mathrm{eV}$. The solid points and curves are calculated from Eqs. (1) and (3), respectively. (b) Bias dependence of the IEC, $-J_{\mathrm{FM}}^{\sigma \sigma^{\prime}}\left(\sigma, \sigma^{\prime}=\uparrow, \downarrow\right)$ in the FM configuration, calculated from Eq. (4), in symmetric $(\delta=0.0 \mathrm{eV})$ MTJ. (c) Energy dependence of $T_{\perp}$ for $\delta=0.0 \mathrm{eV}$ and for $V=-0.2,0$, and $0.2 \mathrm{~V}$, respectively. The blue, black, and red vertical lines refer to $\mu_{R}$, for the above bias values, while $\mu_{L}$ is fixed at $E_{F}=0 \mathrm{eV}$. 


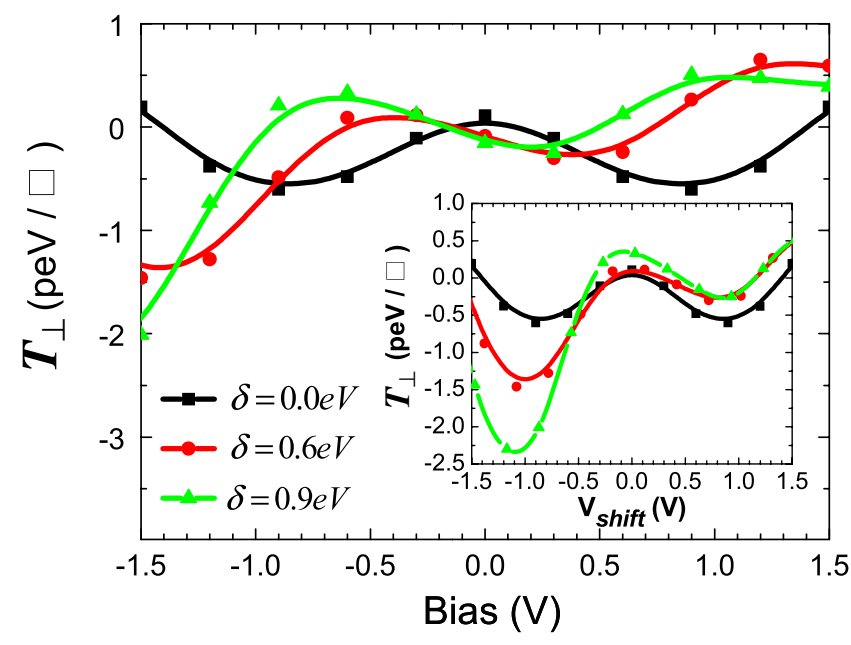

FIG. 4 (color online). High-bias $(|V| \leq 1.5 \mathrm{~V})$ oscillatory behavior of $T_{\perp}(\theta=\pi / 2)$ with $\varepsilon_{L}^{\uparrow}=0.6 \mathrm{eV}$ and $\varepsilon_{L}^{\downarrow}=1.2 \mathrm{eV}$ and for various values of $\delta$. Inset: $T_{\perp}$ versus shifted bias, $V_{\text {shift }}$, where $V_{\text {shift }}=V+c \delta$, with $c=0.7$.

value at zero bias, due to the same BF of the left and right leads. The different curvatures and zero-bias values are due to the different BF of the $\uparrow$-spin and $\downarrow$-spin bands. On the other hand, $J_{\mathrm{FM}}^{\uparrow \downarrow}( \pm V)=J_{\mathrm{FM}}^{\downarrow \uparrow}(\mp V)$, because the majority (minority) bands of the left lead are separated by $\pm \Delta$ compared to the minority (majority) bands of the right lead. This energy splitting is also responsible for their bias shift and the nonpurely quadratic bias dependence. Interestingly, even though each IEC has a complex bias dependence, their algebraic sum has a purely quadratic behavior for $|V| \leq 0.4 \mathrm{~V}$.

In order to understand the bias-induced origin of $T_{\perp}$, we show in Fig. 3(c) the energy dependence of $T_{\perp}$ in symmetric MTJ for $V=-0.2,0.0$, and $0.2 \mathrm{~V}$, respectively. The blue, black, and red vertical lines represent $\mu_{R}$ for the above bias values and $\mu_{L}=0$. The energies that lie within the bias window between $\mu_{L}$ and $\mu_{R}$, denoted by the red (blue) shaded area in Fig. 3(c) under positive (negative) bias, contribute to the current-induced $T_{\perp}$. The positive (negative) bias induces a shift of the zero-bias positive peak of $T_{\perp}$ near $E_{F}$ towards higher (lower) energy and decreases its height. Furthermore, the bias induces a negative dip in the energy range of $\mu_{L}-\mu_{R} \approx \mp V$ near $E_{F}$. This is due to the fact that $T_{\perp}^{R}(E)<0\left[T_{\perp}^{L}(E)<0\right]$ for positive (negative) bias, because the energy states of the right FM lead are higher (lower) than those of the left FM lead. This behavior is analogous to the zero-bias effect of $\mathrm{BF}$ in Fig. 2. Both these effects result in a bias-induced decrease of $T_{\perp}$ and hence $d^{2} T_{\perp}(V) / d V^{2}<0$.

Finally, we investigate the large-bias $(|V| \leq 1.5 \mathrm{~V})$ behavior of the IEC, which has not yet been explored experimentally. As shown in Fig. 4, we predict that $T_{\perp}(\theta=\pi / 2)$ exhibits a symmetric (asymmetric) oscillatory bias behavior for $\delta=0(\delta \neq 0)$, with a period which increases linearly with $\delta$. We find that the bias dependence of $T_{\perp}$ for $\delta \neq 0$ is shifted by $\Delta V=\mp c \delta$ [minus (plus) sign for positive (negative) $\delta$ ] compared to that of the symmetric MTJ. The constant $c$ depends on the majority and minority bands of the left lead ( $c=0.7$ for our parameter choice). The inset displays $T_{\perp}$ versus the shifted bias, $V_{\text {shift }}=V+$ $c \delta$, where all curves exhibit a universal oscillatory bias behavior, but with a different amplitude which depends on the BF asymmetry. The origin of the linear relation of $\Delta V$ with $\delta$ lies on the asymmetry-induced shift of the bias behavior of the IEC, $J_{\mathrm{FM}}^{\sigma \sigma^{\prime}}$, in Fig. 3(b), compared to their symmetric $(\delta=0)$ behavior. Namely, $\Delta V \approx \Delta V^{\Uparrow}+$ $\Delta V^{\Downarrow}-\Delta V^{\downarrow \uparrow}-\Delta V^{\Uparrow \downarrow}$, where $\Delta V^{\Uparrow \uparrow(\downarrow)} \propto-\delta$ and $\Delta V^{\Uparrow \downarrow(\downarrow)} \propto$ $-(\delta \pm \Delta)$, each varying linearly with $\delta$ but with different slopes.

In conclusion, we predict that the fieldlike spin torque in MTJ exhibits an oscillatory bias behavior, which can be tuned selectively via the asymmetry in BF between the FM leads. In the low bias range, this gives rise to a linear or quadratic bias dependence, with positive or negative curvature, including tuning the bias-induced reversal of the IEC, thus reconciling the apparently contradictory experimental results. The underlying mechanism for the nonequilibrium IEC for noncollinear configuration is the interplay of four independent IEC for the majority and minority-spin bands solely in the FM configuration. Each of the IEC can be controlled by changing the splitting between the spin channels of the left and right FM leads. These theoretical predictions may serve as simple guiding rules for future experimental studies of the IEC in MTJ via controlled fabrication of the FM leads.

We thank E. Y. Tsymbal, M. Chshiev, D.C. Ralph, H. Kubota, M. Stiles, and P. Haney for useful conversations. The work at California State University Northridge was supported by NSF-PREM Grant No. DMR-00116566.

[1] J. C. Slonczewski, Phys. Rev. B 39, 6995 (1989).

[2] L. Berger, Phys. Rev. B 54, 9353 (1996).

[3] A. A. Tulapurkar et al., Nature (London) 438, 339 (2005).

[4] G. D. Fuchs et al., Phys. Rev. Lett. 96, 186603 (2006).

[5] J. C. Sankey et al., Nature Phys. 4, 67 (2008).

[6] H. Kubota et al., Nature Phys. 4, 37 (2008).

[7] S. Petit et al., Phys. Rev. Lett. 98, 077203 (2007).

[8] I. Theodonis et al., Phys. Rev. Lett. 97, 237205 (2006).

[9] C. Heiliger and M. D. Stiles, Phys. Rev. Lett. 100, 186805 (2008); P. M. Haney et al., Phys. Rev. B 79, 054405 (2009).

[10] J. Xiao et al., Phys. Rev. B 77, 224419 (2008).

[11] J. C. Slonczewski, Phys. Rev. B 71, 024411 (2005).

[12] A. Kalitsov et al., Phys. Rev. B 79, 174416 (2009).

[13] Z. Li et al., Phys. Rev. Lett. 100, 246602 (2008).

[14] A. Kalitsov et al., J. Appl. Phys. 99, 08 G501 (2006).

[15] I. Theodonis et al., Phys. Rev. B 76, 224406 (2007). 